\title{
SOME IMBEDDING AND NONIMBEDDING THEOREMS FOR $n$-MANIFOLDS $\left({ }^{1}\right)$
}

\author{
BY \\ BEAUREGARD STUBBLEFIELD \\ PART I \\ The Main Theorems
}

Definitions and purpose. Definition 1.1. Space $S_{1}$ is said to be a primitive skew curve of type 1 if $S_{1}$ consists of the points $a, b, c$ and $x, y, z$, and the $\operatorname{arcs} a x, a y, a z, b x, b y, b z, c x, c y$, and $c z$ where different arcs intersect only at their end-points (see Figure 1).

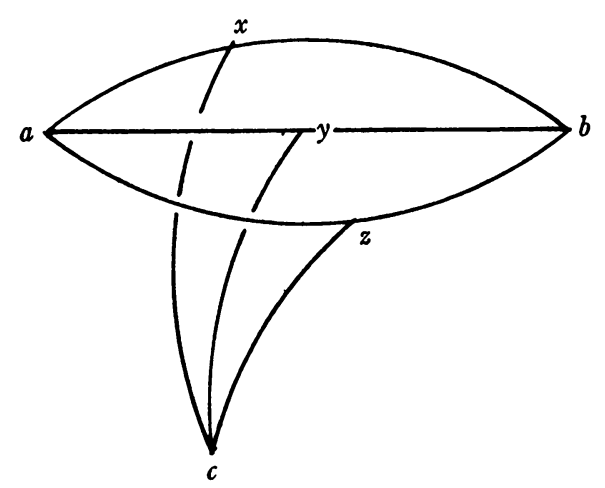

Figure 1. The Primitive Skew Curve, Type 1

Definition 1.2. Space $S_{2}$ is said to be a primitive skew curve of type 2 if $S_{2}$ consists of five distinct points $a, b, c, d$, and $e$ and the arcs $a b, a c, a d, a e$, $b c, b d, b e, c d, c e$, and $d e$, and where different arcs intersect only at their endpoints (see Figure 2).

The first of the two primitive skew curves is the well-known "water, gas, and light problem; the second is "the direct join of five points."

A primitive skew curve $S$ of either type 1 or type 2 is topologically equivalent to no subset of the plane $E^{2}$. Nevertheless, any proper subset of $S$ can be imbedded in $E^{2}$. An immediate consequence is that $S$ is locally imbeddable in the plane. It may be observed that any primitive skew curve

Presented to the Society, April 8, 1961; received by the editors June 30, 1961.

(1) Part of this work was done while the author was a National Science Foundation Research Fellow, NSF Grant G-9652. 


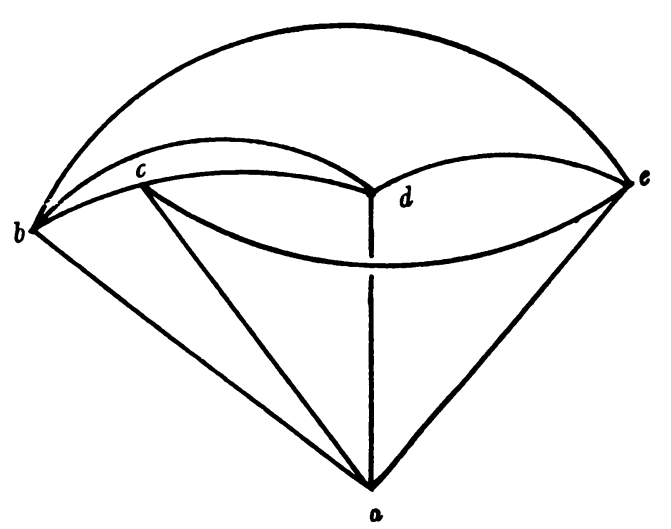

Figure 2. The Primitive Skew Curve, Type 2

can be imbedded in a 2-manifold without difficulty. The curves of Claytor, to be defined subsequently, are also other examples of curves which are homeomorphic with no subset of $E^{2}$. They are not locally imbeddable in the plane and hence cannot be imbedded in a 2-manifold. There are many other examples of one-dimensional Peano continua which have one or more of these properties. Many of them, however, are excluded from this manuscript because they play no important part in these investigations.

Claytor [5] has proved that a necessary and sufficient condition that a Peano continuum be homeomorphic with a subset of $S^{2}$ is that it contains neither a topological image of a primitive skew curve nor a topological image of one of the Claytor curves. It is interesting to investigate the Claytor curves to obtain some of the properties that they have.

The main purpose of this paper is to prove the following:

THEOREM 1.3. If $S$ is a Claytor curve, then for $n$ greater than $1, S \times I^{n-2}$ cannot be imbedded in an n-manifold.

This theorem will be proved separately for each of the two Claytor curves. Since these are the only possible cases, the proof of the Main Theorem will follow immediately.

Throughout this paper, the letter $I$ will denote the closed unit interval $[0,1]$ of the space of real number and $I_{\epsilon}$ will denote the closed subinterval of $I$ having 0 and $\epsilon$ as end-points.

In general, small letters will represent Euclidean points and capital letters will represent sets of points. In particular, the letters $A, J, L, R$ and $D$, with their subscripts, represent arcs, Jordan curves, line segments, radii and diameters of circles, respectively. Exceptions are the letters $i, j, n, m, N$ and $M$, which denote natural numbers. Small Greek letters stand for real numbers with $\pi$ the metric on $E^{n}$. The symbols $\phi, S^{n}$, and $\theta(W)$ or $W^{\prime}$ will respectively 


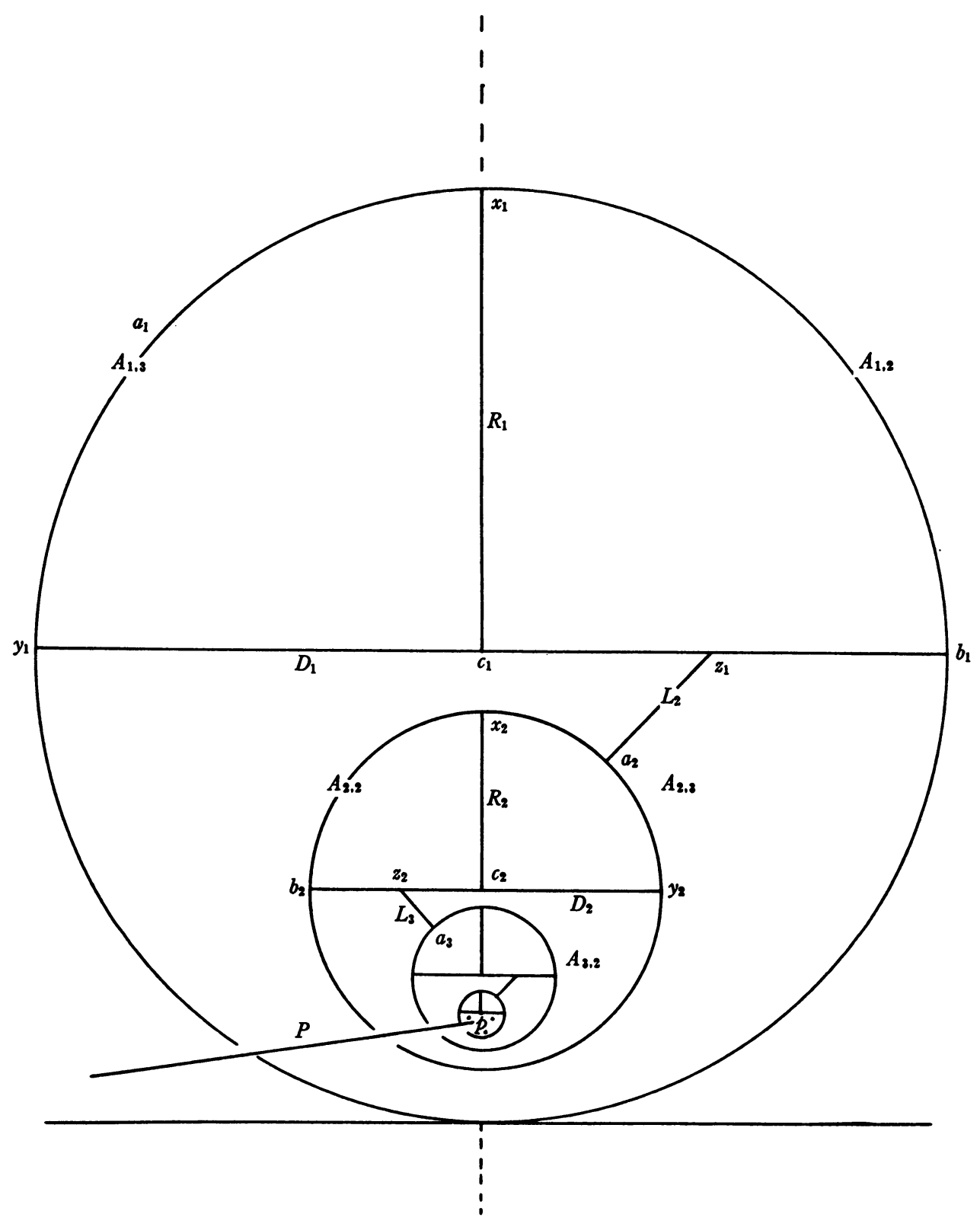

Figure 3. The First Claytor Curve

represent the empty set, the $n$-sphere and the homeomorphic image of the set $W$. Capital script letters designate open sets homeomorphic with $\mathfrak{T}^{n}$. 
Specifically, $\mathcal{S}^{n}$ is an $n$-dimensional spherical neighborhood. $\mathfrak{T}^{n}$ is an $n$ manifold.

Definition 1.4. For any positive integer $n$, let

(a) $J_{n, 1}=a_{n} x_{n} b_{n} y_{n} \subset E^{3}$ be the points $(\xi, \eta, \zeta)$ such that $\zeta=0$ and $\xi^{2}+\left(\eta-\eta_{n}\right)^{2}=\left(\rho_{n}\right)^{2}$ where $\eta_{1}=\rho_{1}=1 / 2$ and when given $\eta_{n}$ and $\rho_{3}, \eta_{n+1}=\eta_{n}$ $-\rho_{n} / 2$ and $\rho_{n+1}=3 / 4\left(\eta_{n+1}-\eta_{n}+\rho_{n}\right)$;

(b) $A_{n, 2}=x_{n} b_{n} \subset J_{n, 1}$ be the points such that $\eta \geqq \eta_{n}$ and $(-1)^{n} \xi \leqq 0$;

(c) $A_{n, 3}=x_{n} a_{n} y_{n} \subset J_{n, 1}$ be the points such that $\eta \geqq \eta_{n}$ and $(-1)^{n} \xi \geqq 0$;

(d) $A_{n, 4}=b_{n} y_{n} \subset J_{n, 1}$ be the points such that $\eta \leqq \eta_{n}$;

(e) $R_{n}=x_{n} c_{n}$ be all points $(\xi, \eta, \zeta)$ such that $\eta_{n} \leqq \eta \leqq \eta_{n}+\rho_{n}, \xi=\zeta=0$;

(f) $p$ be the limit of $r_{n}, r_{n}$ in $R_{n}$, as $n$ increases;

(g) $D_{n}=y_{n} c_{n} z_{n} b_{n}$ be all points $(\xi, \eta, \zeta)$ such that $\zeta=0, \eta=\eta_{n}$ and $|\xi| \leqq \rho_{n}$;

(h) $L_{n+1}=z_{n} a_{n+1}$ be the line segment connecting the midpoint of $A_{n+1,3}$ to $\left(-1 / 2(-1)^{n} \rho_{n}, \eta_{n}, 0\right)$; and

(i) $P$ be a sticker at $p$.

The set $S$ consisting of all points of the sets above is the first Claytor curve (see Figure 3).

Definition 1.5. For each integer $i$, the points $a_{i}$ and $p$ of $S$ separate $S$. We define $B_{i}$ to be the closure of that component containing $c_{i}$. Each such $B_{i}$ is referred to as "a base of the curve."

In this section, we prove the following

THEOREM 1.6. Let $S$ be the first of the Claytor curves. Then, for $n$ greater than 1, $Q=S \times I^{n-2}$ cannot be imbedded in $E^{n}$.

It is sufficient to prove the theorem for $n \neq 2$ since the theorem for the case $n=2$ follows immediately from the case $n=3$.

We suppose the contrary and let $\theta$ be a function defining the homeomorph of $S \times I^{n-2}$ in $E^{n}$. Let $p^{\beta}=\theta(p \times \beta \times 0 \times \cdots \times 0), 0 \leqq \beta \leqq 1$, and let $\pi\left(p^{0}, p^{1}\right)$ $=\pi_{0}$. By uniform continuity, there is a positive number $\epsilon$ such that for $r, s$ in $Q$ such that $\pi(r, s) \leqq \epsilon$, then $\pi\left(r^{\prime}, s^{\prime}\right)$ is less than $\pi_{0} / 4(n-2)$. We need only prove that $Q_{\epsilon}$, the cross product of $S$ and $I \times I_{\epsilon}^{n-3}$, cannot be imbedded in $E^{n}$ under $\theta$.

Let $p_{1}, p_{2}, p_{3}$ be the three points, in that order, which divide the line segment $L$ from $p^{0}$ to $p^{1}$ into four equal parts such that

$$
\pi\left(p^{0}, p_{1}\right)=\pi\left(p_{1}, p_{2}\right)=\pi\left(p_{2}, p_{3}\right)=\pi\left(p_{3}, p^{1}\right)=\pi_{0} / 4
$$

Let $\Pi_{i}, i=1,2,3$, be the $(n-1)$-hyperplanes perpendicular to $L$ at points $p_{i}$ with $\Pi_{2}$ having a point $p^{\beta_{0}}$ in common with the image of the cross product of $p$ and $I \times I_{e}^{n-3}$. Let $N$ be the smallest positive integer such that $J_{N, 1}$ is of diameter less than $\epsilon$. It is certainly true that for any point $p_{0}$ in $B_{i}, i \geqq N$, (see Definition 1.5), $\theta\left[p_{0} \times 0 \times I_{e}^{n-3}\right]$ is below the three hyperplanes and $\theta\left[p_{0} \times 1 \times I_{e}^{n-3}\right]$ is above these planes. For any subset $W$ of $S$, all of whose points are within $\epsilon$ of $p$, we define $[W]^{-}$as that component of $\theta\left(W \times I \times I_{\epsilon}^{n-3}\right)-\Pi_{1}-\Pi_{3}$ which 
contains $\theta\left[W \times \beta_{0} \times I^{n-3}\right]$. Also, for a positive integer $n$, let

$$
\begin{aligned}
& J_{n, 2}=A_{n, 2}+R_{n}+\left[(\xi, \eta, \zeta) \text { in } D_{n} \text { such that }(-1)^{n} \xi \leqq 0\right], \\
& J_{n, 3}=A_{n, 3}+R_{n}+\left[(\xi, \eta, \zeta) \text { in } D_{n} \text { such that }(-1)^{n} \xi \geqq 0\right], \\
& J_{n, 4}=A_{n, 4}+D_{n}
\end{aligned}
$$

for each $i \geqq N$, and each $j=1,2,3,4$, we let ${ }_{j} \mathfrak{U}_{i}$ be the bounded component of $V-\left[J_{i, j}\right]-$ where $V$ is the space between the two planes $\Pi_{1}$ and $\Pi_{3}$ separated by $\left[J_{i, j}\right]-$. For any $i \geqq N$, there exists a 1 -to- 1 correspondence between the separations of $V$ by $\left[J_{i, j}\right]-$ and the separations of the $\xi, \eta$-plane by $J_{i, j}$.

LEMma 1.7. If for some $i, i \geqq N$ and some $j(j=1,2,3,4),{ }_{j} \mathfrak{u}_{i}$ contains some point of $\left[B_{i}\right]^{-}$(see Definition 1.5), then ${ }_{j} \mathcal{u}_{i}$ contains all points of $\left[B_{i}\right]^{-}$.

Proof. Since $\left[J_{i, j}\right]^{-}$separates $V$ and cannot possibly separate $\left[B_{i}\right]^{-}$, then ${ }_{j} \mathcal{U}_{i}$ necessarily contains all points of $\left[B_{i}\right]-$ if it contains any one point of $\left[B_{i}\right]^{-}$.

Lemma 1.8. For some $i, i \geqq N$, and some $j(j=1,2,3,4),{ }_{j} \mathcal{u}_{i}$ does contain a point of $\left[B_{i}\right]-$ of Lemma 1.7 .

Proof. Suppose the contrary. That is, for all $i \geqq N,{ }_{j} \mathcal{U}_{i} \cdot\left[B_{i}\right]^{-}=\phi$ for each $j$.

For any $i$, by construction, $J_{i, 3} \cdot L_{i+1} \neq \phi$. For each $j,{ }_{3} \mathcal{U}_{i}$ necessarily contains no point of $\left[J_{i, j}\right]^{-}$and, hence, no point of $\left[B_{i}\right]^{-}$(see Figure 4).

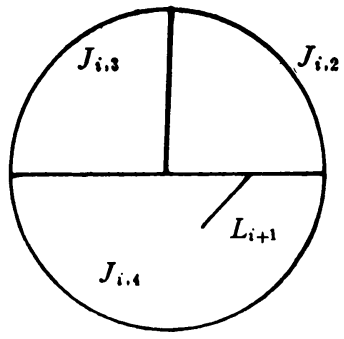

FIGURE 4. $J_{i, 2}, J_{i, s}$, and $J_{i, 4}$ with $L_{i+1}$

Case 1. ${ }_{2} \boldsymbol{u}_{i}$ contains $\left(\left[J_{i, 1}\right]^{-}+\left[J_{i, 3}\right]^{-}\right)-\left[J_{i, 2}\right]^{-}$. We note that if ${ }_{2} \mathcal{u}_{i}$ contains any point of this set, then it contains all such points. Here, either ${ }_{3} \mathcal{U}_{i+1}$ contains $\left[B_{N}\right]^{-}-\left[B_{i}\right]^{-}$or ${ }_{1} \mathfrak{u}_{i+1}$ contains $\left[B_{N}\right]^{-}-\left[B_{i}\right]^{-}$(see Figure 5). In each case, it is true that for some $j$ for $j=1,2,3$, or $4, j \mathcal{U}_{i+1}$ contains $\left[B_{N}\right]^{-}-\left[B_{i}\right]^{-}$.

Case 2. Neither ${ }_{2} \mathcal{U}_{i}$ nor ${ }_{3} \mathcal{U}_{i}$ contains either a point of $\left[J_{i, j}\right]^{-}$or a point of $\left[B_{i}\right]^{-}$, for any $j$. Then, ${ }_{1} \mathcal{U}_{i+1}$ contains all of $\left[B_{N}\right]^{-}-\left[B_{i}\right]^{-}$. Again, we obtain the result that for some $j(j=1,2,3,4),{ }_{j} u_{i+1}$ contains $\left[B_{N}\right]^{-}-\left[B_{i}\right]-$ (see Figure 6).

Cases 1 and 2 of Lemma 1.8 holds for any $i \geqq N$ and no other possible cases 

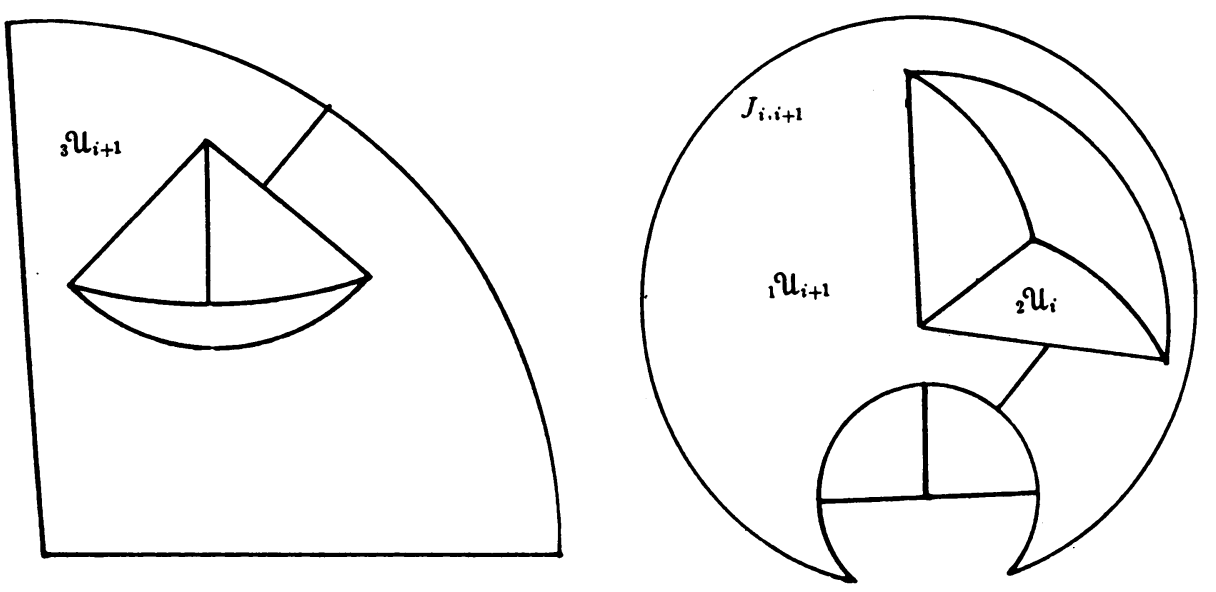

Figure 5. Case 1 of Lemma 1.8

exist. There exists a sequence $n_{i}$ with each $n_{i}$ having value $1,2,3$, or 4 such that for $i>N, n_{i} u_{i}$ contains $\left[B_{N}\right]^{-}-\left[B_{i}\right]^{-}$. The $\left[J_{i, n_{i}}\right]^{-}$form a sequence of nonintersecting but expanding sets each of which separates $v$. Let $J$ be the limit of the $\left[J_{i, n_{i}}\right]-$. Take $x_{0}$ in $\mathfrak{u}_{N}={ }_{n_{N}} \mathfrak{u}_{N} C_{n_{i}} \mathfrak{u}_{i}$ for all $i>N$. By compactness, we have $\pi_{0}=\max \pi\left(x_{0}, x\right)$ for all $x$ in $Q^{\prime}$. Take $x_{i}$ in $v$ such that $\pi\left(x_{0}, x_{1}\right)$

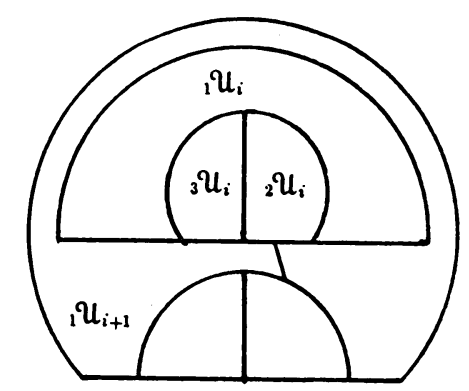

Figure 6. Case 2 of Lemma 1.8

$=2 \pi_{0}$. Let $A$ be any arc in $v$ connecting $x_{0}$ and $x_{1}$. Since, for $i>N,\left[J_{i, n_{i}}\right]^{-}$ separates $A$, the $\left[J_{i, n_{i}}\right]-$ contains a point $a_{i}$ which is a relatively interior point of $A$. Let the limit of the $a_{i}$ be $q$ in $J$. Also, $q$ is in $A$ since $A$ is itself compact. We have $\pi\left(q, x_{1}\right) \geqq \pi_{0}$ and $q$ is not in the set $u_{N}$. Neither $x_{0}$ nor $x_{1}$ is in $J$. Hence, $x_{0}$ and $x_{1}$ are separated in $A$ by $J$. Since $A$ was arbitrarily chosen, it follows that $V$ is separated by $J$. But this gives rise to a contra- 
diction since the limit under the homeomorphism is $[p]-$ which is $(n-2)$ dimensional and cannot possibly separate $V$. Thus, it is true that for some $i, i \geqq N$, and some $j(j=1,2,3,4),{ }_{j} u_{i}$ does contain a point of $\left[B_{i}\right]-$ of Lemma 1.7 .

LEMMA 1.9. If for some positive integer $i$ and for some $j$, where $j=1,2,3,4$, ${ }_{j} u_{i}$ contains a point of $\left[B_{i}\right]^{-}$of Lemma 1.7 , then, for some $j,{ }_{j} u_{i+1} \cdot\left[B_{i+1}\right]^{-} \neq \phi$.

Proof. Case 1. $\mathcal{u}_{i}$ contains a point of $\left[B_{i}\right]^{-}$. By Lemma 1.7, it contains all points of $\left[B_{i}\right]^{-}$. Then, either ${ }_{2} \mathcal{U}_{i}$ or ${ }_{4} \mathfrak{U}_{i}$ contains $\left[B_{i}\right]^{-}$(see Figure 7 ).
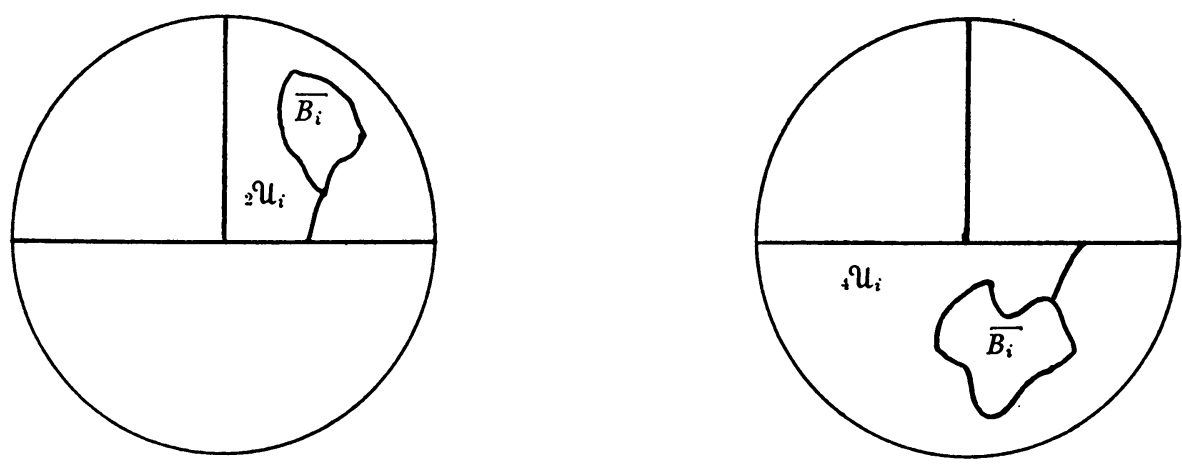

Figure 7. Case 1 of Lemma 1.9

In each subcase ${ }_{1} \mathcal{U}_{i+1}$ contains a point of $\left[B_{i+1}\right]^{-}$.

Case 2. The results of Case 1 are obtained if ${ }_{3} \mathcal{u}_{i}$ contains a point of $\left[B_{i}\right]^{-}$(see Figure 8).
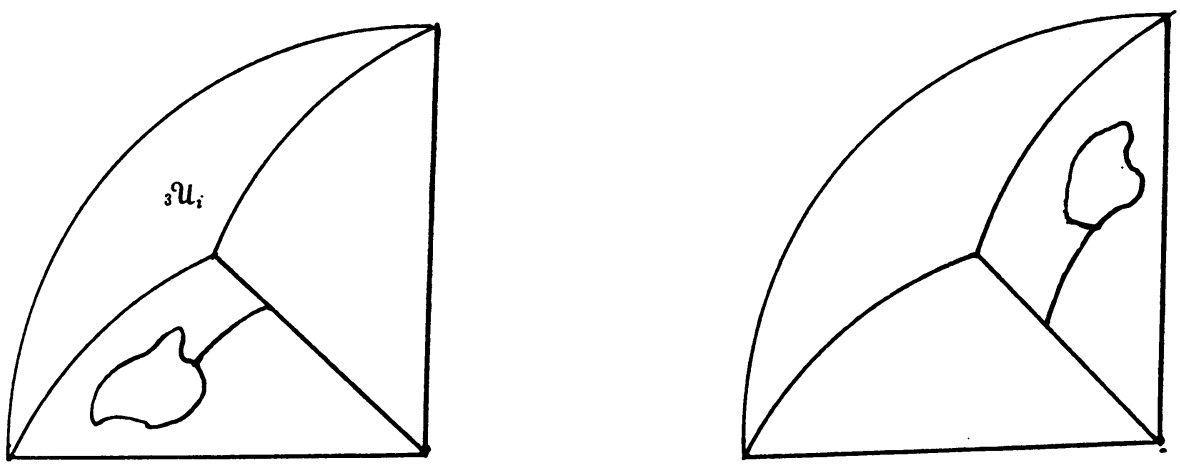

FIGURE 8. Case 2 of Lemma 1.9 
Case 3. ${ }_{2} \mathcal{U}_{i}$ contains a point of $\left[B_{i}\right]-$. By Lemma 1.7 , it contains all points of $\left[B_{i}\right]^{-}$. Here, ${ }_{1} \mathfrak{u}_{i+1}$ and/or ${ }_{4} \mathfrak{u}_{i+1}$ contains $\left[B_{i+1}\right]^{-}$.
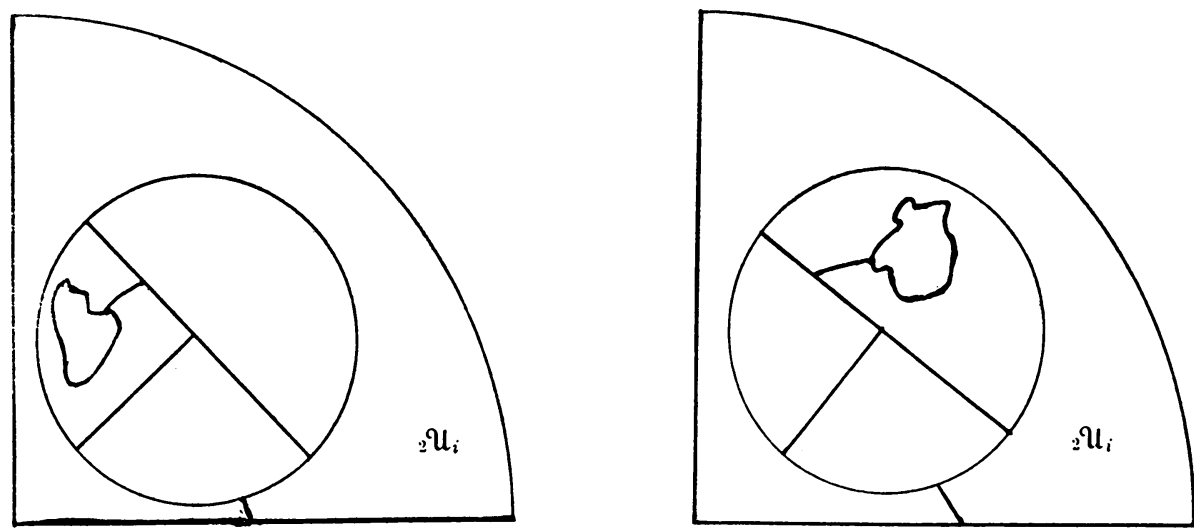

Figure 9. Case 3 of Lemma 1.9

Case 4. The results of Case 3 are the same if ${ }_{4} u_{i}$ contains a point of $B_{i}$.
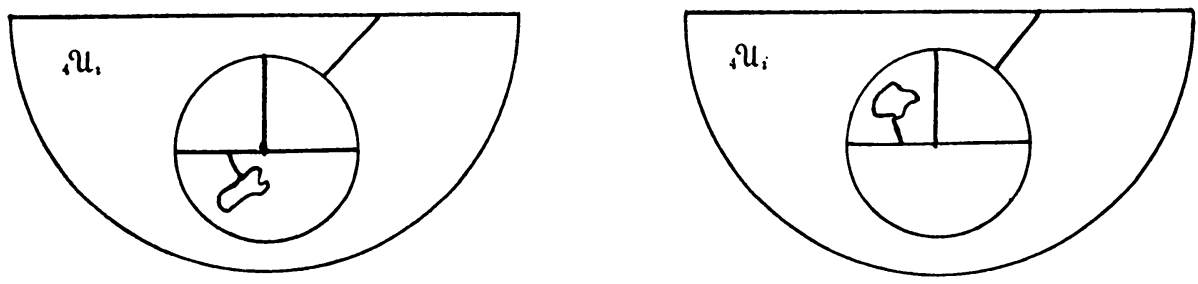

FIGURE 10. Case 4 of Lemma 1.9

Lemma 1.10. There exists a sequence $n_{i}, i$ a positive integer, $n_{i}=1,2,3$ or 4 , such that for some positive integer $M$,

$$
\bigcap_{i=M}^{\infty}{ }_{n_{i}} \mathcal{u}_{i} \supset[p]^{-} \text {. }
$$

Proof. We take $M$ from Lemma 1.8 and $n_{i}$ from Lemma 1.9 and apply Lemma 1.7.

THEOREM 1.6. Let $S$ be the first of the Claytor curves. Then, $S \times I^{n-2}, n \geqq 2$, cannot be imbedded in $E^{n}$.

Proof. Suppose the contrary. Let $\theta$ be our homeomorphic map. By Lemma 1.10 , we obtain a sequence $n_{i}$ such that ${ }_{n_{i}} \mathfrak{u}_{i}$ contains $[p]^{-}$for all $i$ greater than some integer $N_{1}$. Let $\delta^{n}$ be a spherical neighborhood about $p^{\beta_{0}}$ of diam- 
eter $\pi_{0}=$ minimum of the distance of $p^{\beta_{0}}$ to the boundary of $\left(S \times I^{n-2}\right)^{\prime}$ and $\pi_{0} / 4$. Let $T$ be any arc in $S^{n} \cap\left(P \times I^{n-2}\right)^{\prime}$ having exactly the point $p^{\beta_{0}}$ in common with $[p]^{-}$. Take $q$, any point on $T$ distinct from $p^{\beta_{0}}$. Let $\pi_{2}$ be the distance from $p^{\beta_{0}}$ to $q$. By uniform continuity, there exists an $N_{2}$ such that $\pi\left([p]^{-},\left[J_{i, n_{i}}\right]^{-}\right)<\pi_{2}$ for all $i \geqq N_{2}$. Here, we take $N$ to be the maximum of $N_{1}$ and $N_{2}$. Then, $J=\left[J_{N, n_{N}}\right]-$ separates $V$ and $T$ by the Alexander Duality Theorem. Hence, $T$ has a point in common with $J$. This gives rise to a contradiction.

DEFinition 1.11. For any positive integer $n$, let

(a) $J_{n, 1}=a_{n} b_{n} d_{n}$ be the subset of $E^{3}$ consisting of all points $(\xi, \eta, \zeta)$ such that $\zeta=0$ and $\xi^{2}+\left(\eta-\eta_{n}\right)^{2}=\left(\rho_{n}\right)^{2}$ where $\eta_{1}=\rho_{1}=1 / 2$, and when given $\eta_{n}$ and $\rho_{n}, \eta_{n+1}=\eta_{n}-\rho_{n} / 2$ and $\rho_{n+1}=3 / 4\left(\eta_{n+1}-\eta_{n}+\rho_{n}\right)$;

(b) $D_{n}=b_{n} e_{n} d_{n}$ be all $(\xi, \eta, \zeta)$ such that $\eta=\eta_{n},|\xi| \leqq \rho_{n}, \zeta=0$;

(c) $p$ be the limit of $r_{n}, r_{n}$ in $D_{n}$, as $n$ increases;

(d) $T$ be the line segment from $p$ to the point $(0,1,0)$;

(e) $A_{n}=b_{n} c_{n} d_{n}$ be the points $(\xi, \eta, \zeta)$ such that $\eta \geqq \eta_{n}, \zeta=0$ and $\xi^{2}+\left(\eta-\eta_{n+1}\right)^{2}$ is equal to $\left(\eta_{n+1}-\eta_{n}\right)^{2}+\left(\rho_{n}\right)^{2}$;

(f) $P$ be a sticker at $p$;

(g) $A_{n, 2}=b_{n} c_{n}$ be the subset of $A_{n}$ such that $\xi \leqq 0$;

(h) $A_{n, 3}=c_{n} d_{n}$ be the subset of $A_{n}$ such that $\xi \geqq 0$;

(i) $A_{n, 4}=b_{n} d_{n}$ be the subset of $J_{n, 1}$ such that $\eta \leqq \eta_{n}$;

(j) $R_{n}=a_{n} c_{n} e_{n}$ be the subset of $T$ such that $\eta_{n} \leqq \eta \leqq\left(\eta_{n+1}-\eta_{n}\right)^{2}+\left(\rho_{n}\right)^{2}$ $+\eta_{+1} ;$ and

(k) $L_{n+1}=e_{n} a_{n+1}$ be the points $(0, \eta, 0)$ such that $\eta_{n+1}+\rho_{n} \leqq \eta \leqq \eta_{n}$.

'The set $S$ consisting of all the points of the sets above is, by definition, the second Claytor curve (see Figure 11).

In this section, we shall prove the following

TheOREM 1.12. Let $S$ be the second Claytor curve. Then, for $n$ greater than 1 , $Q=S \times I^{n-2}$ cannot be imbedded in $E^{n}$.

It is sufficient to prove the theorem for $n \neq 2$ since the theorem for the case $n=2$ follows immediately from the case $n=3$. Let us suppose that the theorem is false and that there exists some homeomorphic function $\theta$ sending $S \times I^{n-2}$ into $E^{n}$. For any $\beta$ in $I$, let $p^{\beta}=\theta(p \times \beta \times 0 \times \cdots \times 0)$, and let $\pi\left(p^{0}, p^{1}\right)=\pi_{0}$. Here, we use the fact that $\theta$ is necessarily uniformly continuous. There is a positive number $\epsilon$ such that for $r, s$ in $Q, \pi(r, s) \leqq \epsilon$ implies that $\pi\left(r^{\prime}, s^{\prime}\right)$ is less than $\pi_{0} / 4(n-2)$. We need only show that $Q_{\epsilon}$, the cross product of $S$ and $I \times I_{\epsilon}^{n-3}$, cannot be imbedded in $E^{n}$ under the function $\theta$.

Let $p_{1}, p_{2}, p_{3}$ be the three points, in that order, which divide line segment $L$ from $p^{0}$ to $p^{1}$ into four equal parts such that

$$
\pi\left(p^{0}, p_{1}\right)=\pi\left(p_{1}, p_{2}\right)=\pi\left(p_{2}, p_{3}\right)=\pi\left(p_{3}, p^{1}\right)=\pi_{0} / 4 \text {. }
$$

Let $\Pi_{i}, i=1,2,3$, be the $(n-1)$-hyperplanes perpendicular to $L$ at points $p_{i}$ 


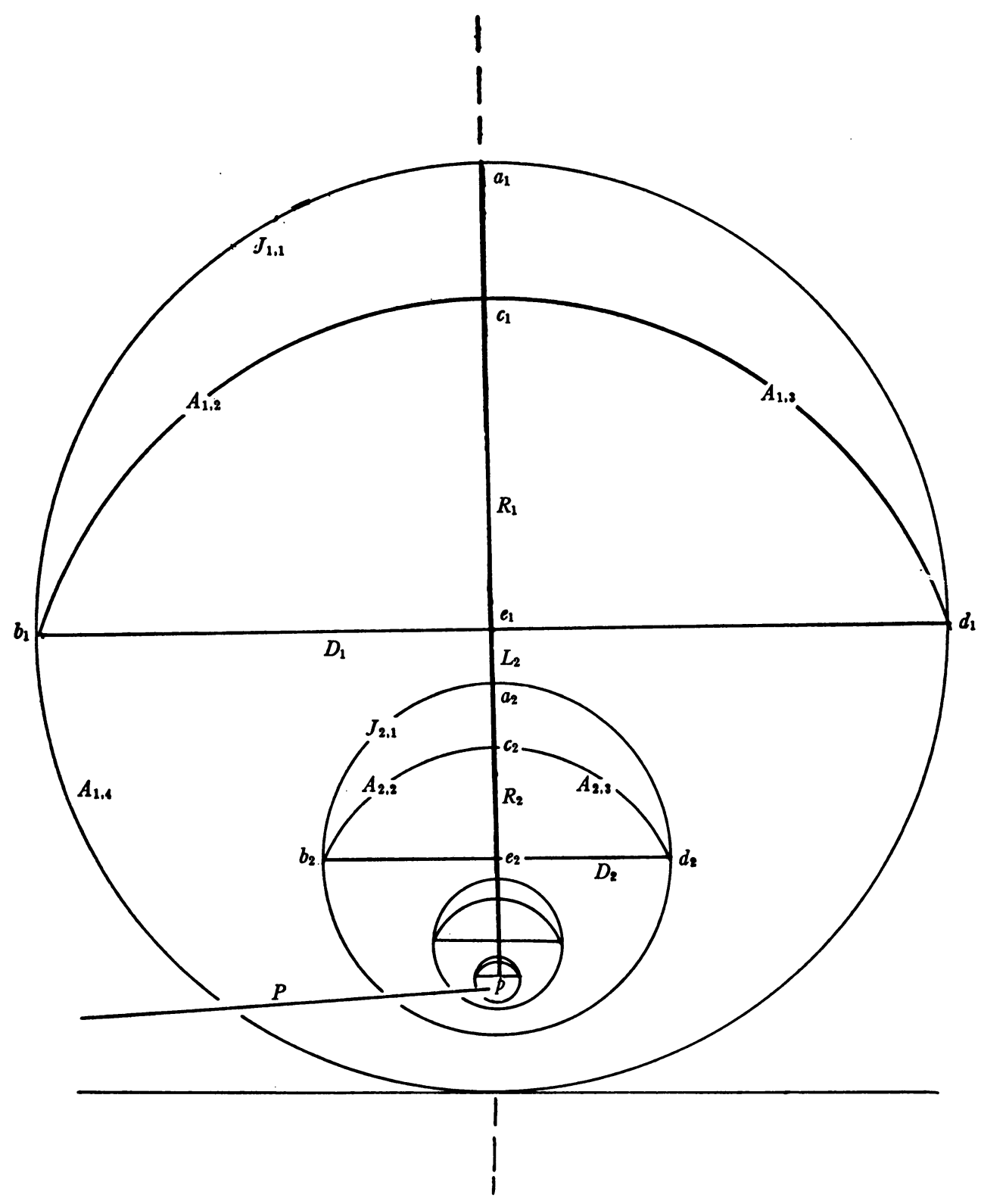

Figure 11. The Second Claytor Curve

with $\Pi_{2}$ having a point $p^{\beta_{0}}$ in common with the image of the cross product of $p$ and $I \times I_{a}^{n-3}$. Let $N$ be the smallest positive integer for $J_{i, 1}$ to be of diameter less than $\epsilon$ for all $i \geqq N$. For $i \geqq N$, and $p_{0}$ in $B_{i}$ (see Definition 1.5), 
$\theta\left[p_{0} \times 0 \times I_{e}^{n-3}\right]$ is below the three hyperplanes and $\theta\left[p_{0} \times 1 \times I_{e}^{n-3}\right]$ is above these planes. For any subset $W$ of $S$, all of whose points are within $\epsilon$ of $p$, we define $[W]^{-}$to be that component of $\left(W \times I \times I_{c}^{n-3}\right)^{\prime}-\Pi_{1}-\Pi_{3}$ which contains $\theta\left[W \times \beta_{0} \times I_{e}^{n-3}\right]$. Also, for any positive integer $n$, let

$$
\begin{aligned}
& J_{n, 2}=A_{n, 2}+R_{n}+\left[(\xi, \eta, \zeta) \text { in } D_{n} \text { such that }(-1)^{n} \xi \leqq 0\right], \\
& J_{n, 3}=A_{n, 3}+R_{n}+\left[(\xi, \eta, \zeta) \text { in } D_{n} \text { such that }(-1)^{n} \xi \geqq 0\right], \\
& J_{n, 4}=A_{n, 4}+D_{n}, \text { and }
\end{aligned}
$$

for each $i \geqq N$, and each $j=1,2,3,4$, we let ${ }_{j} \mathcal{u}_{i}$ be the bounded component of $V-\left[J_{i, j}\right]-$ where $V$ is the space between the two planes $\Pi_{1}$ and $\Pi_{3}$ separated by $\left[J_{i, j}\right]-$. For any $i \geqq N$, there exists a 1-to-1 correspondence between the separations of $U$ by the $\left[J_{i, j}\right]-$ and the separations of the plane by the $J_{i, j}$.

LEMмA 1.13. If for some $i, i \geqq N$, and some $j(j=1,2,3,4),{ }_{j} \mathfrak{u}_{i}$ contains some point of $\left[B_{i}\right]^{-}$, then ${ }_{j} \mathcal{U}_{i}$ contains all points of $\left[B_{i}\right]^{-}$.

Proof. Since $\left[J_{i, j}\right]^{-}$separates $\mathcal{V}$ and cannot possibly separate $\left[B_{i}\right]^{-}$, then ${ }_{j} \mathcal{U}_{i}$ necessarily contains all points of $\left[B_{i}\right]-$ if it contains any one point of $\left[B_{i}\right]^{-}$.

Lemma 1.14. For some $i, i \geqq N$, and some $j(j=1,2,3,4)$,

$$
j u_{i} \cdot\left[B_{i}\right]-\neq \phi .
$$

Proof. Suppose the contrary. That is, for all $i \geqq N$ and for each $j(j=1,2$, 3 , or 4)

$$
j u_{i} \cdot\left[B_{i}\right]-=\phi .
$$

For $i \geqq N,{ }_{1} \mathcal{U}_{i}$ necessarily contains no point of $\left[J_{i, j}\right]-$ (for each $j$ ) and no point of $\left[B_{i}\right]-$ (see Figure 12).

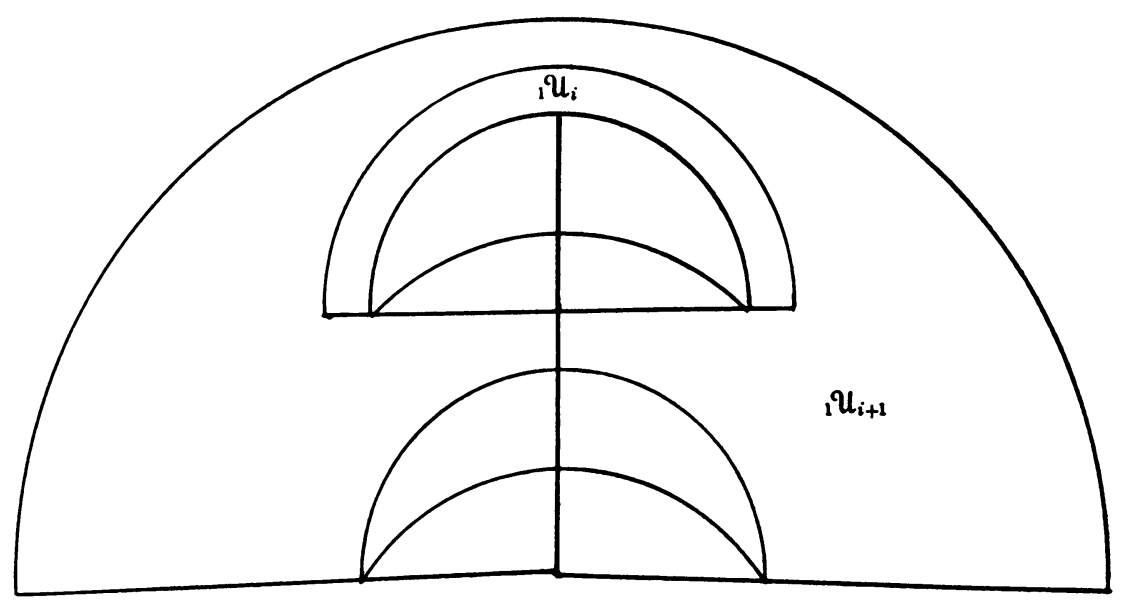

FIGURE 12. ${ }_{1} U_{i}$ and $\left[J_{i, j}\right]^{-}$of Lemma 1.14 
We note that if ${ }_{1} \mathfrak{u}_{i}$ contains any point of this set, then it contains all such points and we get a contradiction.

Case 1. ${ }_{2} \mathfrak{u}_{i}$ contains $\left(\left[J_{i, 1}\right]^{-}+\left[J_{i, 3}\right]^{-}\right)-\left[J_{i, 2}\right]^{-}$. Then some ${ }_{j} \mathfrak{u}_{i+1}$ contains $\left[B_{N}\right]^{-}-\left[B_{i}\right]^{-}$(see Figure 13 ).
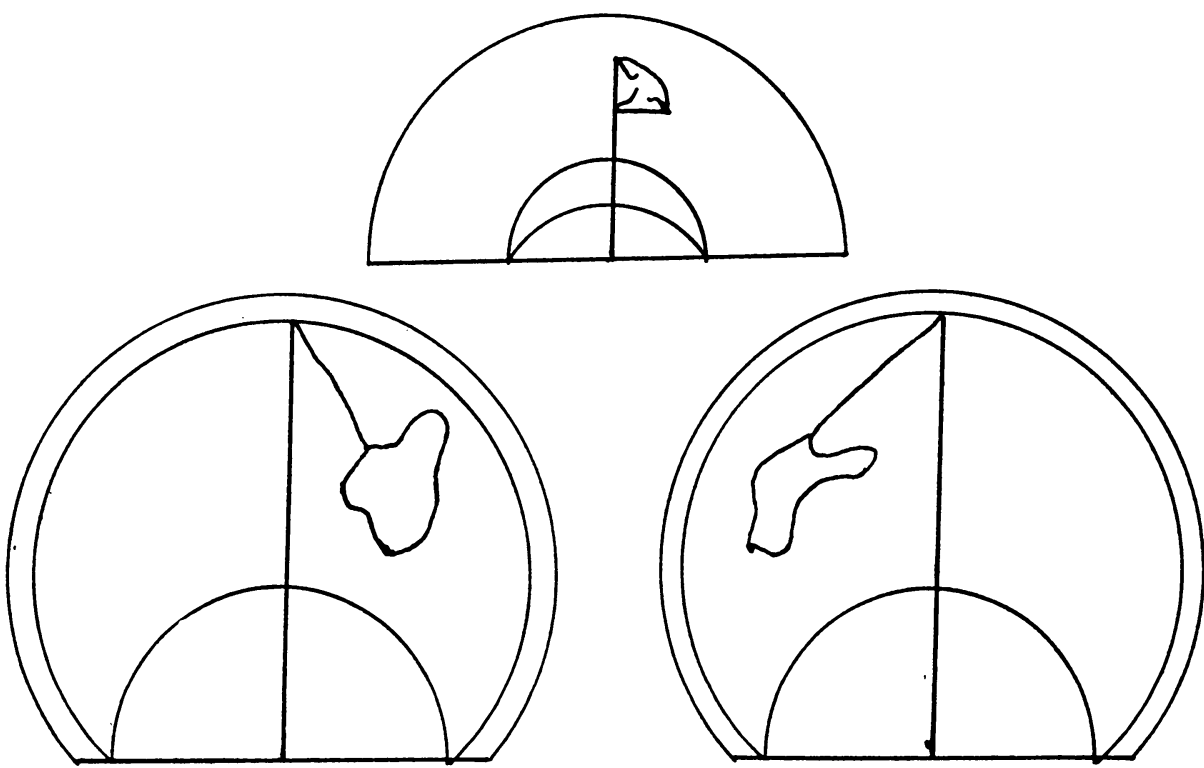

FIGURE 13. Case 1 of Lemma 1.14

Case 2. ${ }_{3} \mathcal{U}_{i}$ contains $\left(\left[J_{i, 1}\right]^{-}+\left[J_{i, 2}\right]^{-}\right)-\left[J_{i, 3}\right]-$. Then some ${ }_{j} \mathfrak{u}_{i+1}$ contains $\left[B_{N}\right]^{-}-\left[B_{i}\right]^{-}$(see Figure 14).
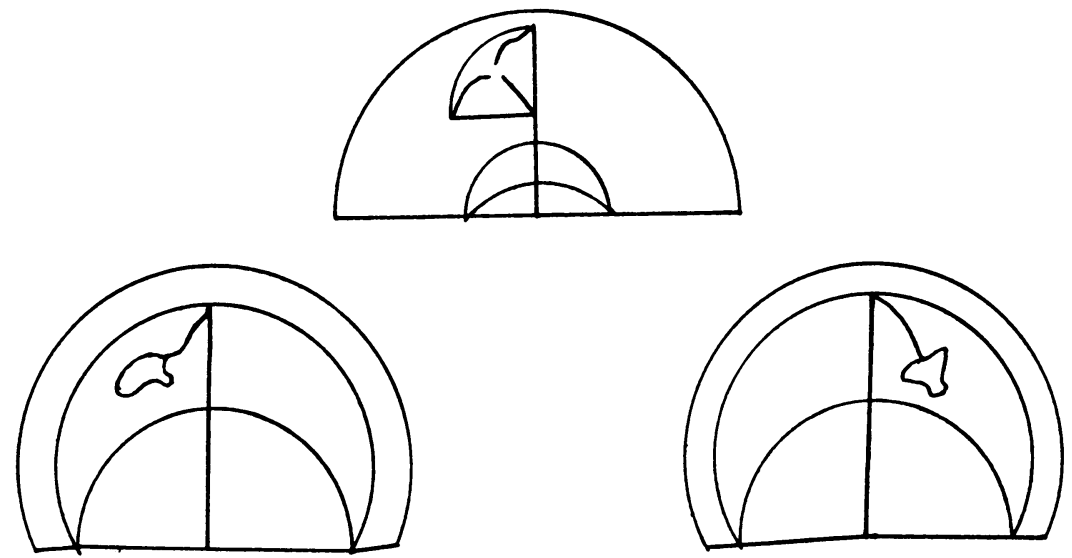

Figure 14. Case 2 of Lemma 1.14 
Case 3. Neither Case 1 nor Case 2 occurs. Then some ${ }_{j} \mathfrak{u}_{i+1}$ contains $\left[B_{N}\right]^{-}-\left[B_{i}\right]^{-}$.

Since it is true that in each case, for some $j,{ }_{j} u_{i+1}$ contains $\left[B_{N}\right]--\left[B_{i}\right]-$ for all $i \geqq N$, then there exists a sequence $n_{i}, n_{i}$ having values $1,2,3$, or 4 such that for $i>N, n_{i} \mathcal{u}_{i}$ contains $\left[B_{N}\right]^{-}-\left[B_{i}\right]^{-}$. The $\left[J_{i+1, n_{i}}\right]^{-}$form a sequence of nonintersecting but expanding sets each of which separates $V$ and whose limit separates $v$. But this is impossible since the limit under the homeomorphism is $[p]^{-}$which is $(n-2)$-dimensional and cannot possibly separate $v$.

LemMa 1.15. If for some positive integer $i$ and some $j$ where $j=1,2,3$ or 4 , ${ }_{j} \mathcal{u}_{i}$ contains a point of $\left[B_{i}\right]^{-}$of Lemma 1.13 , then, for some $j,{ }_{j} u_{i+1} \cdot\left[B_{i+1}\right]^{-}$ $\neq \phi$.

Proof. Case 1. ${ }_{1} \mathcal{U}_{i}$ contains a point of $\left[B_{i}\right]-$. By Lemma 1.13, ${ }_{1} \mathfrak{U}_{i}$ contains all points of $\left[B_{i}\right]-$. Then one of ${ }_{2} \mathfrak{u}_{i},{ }_{3} \mathfrak{u}_{i}$ and ${ }_{4} \mathfrak{u}_{i}$ contains $\left[B_{i}\right]-$. In each subcase, ${ }_{1} \mathcal{U}_{i+1}$ contains a point of $\left[B_{i+1}\right]-$. By Lemma 1.13 it contains all of $\left[B_{i+1}\right]^{-}$(see Figure 15).
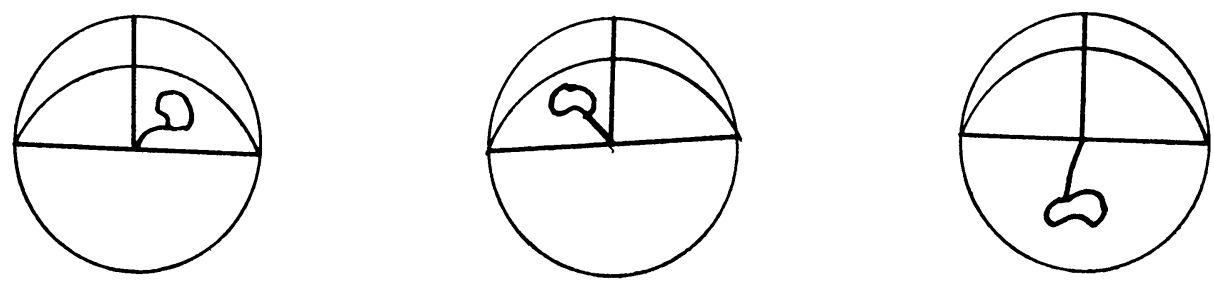

FIGURE 15. Case 1 of Lemma 1.15

Case 2. ${ }_{2} \mathcal{U}_{i}$ contains a point of $\left[B_{i}\right]^{-}$. Then ${ }_{1} \mathcal{u}_{i+1}$ contains $\left[B_{i+1}\right]^{-}$. Case 3. ${ }_{3} \mathcal{U}_{i}$ contains a point of $\left[B_{i}\right]^{-}$. Then ${ }_{1} \mathcal{U}_{i+1}$ contains $\left[B_{i+1}\right]^{-}$. Case 4 . ${ }_{4} \mathfrak{U}_{i}$ contains a point of $\left[B_{i}\right]^{-}$. Then ${ }_{1} \mathfrak{U}_{i+1}$ contains $\left[B_{i+1}\right]-$. See Figure 16 for Cases 2, 3 and 4.

Lemma 1.16. There exists a sequence $n_{i}$, where $n_{i}=1, \cdots, 4$ for each $i$, such that for some $M, \bigcap_{i=M}^{\infty} n_{i} \mathcal{U}_{i}=[p]^{-}$.

Proof. We take $M$ from Lemma 1.14, $n_{i}$ from Lemma 1.15 and apply Lemma 1.13.

Theorem 1.12. Let $S$ be the second of the Claytor curves. Then, $S \times I^{n-2}$, $n \geqq 2$, cannot be imbedded in $E^{n}$.

Proof. Suppose the contrary. Let $\theta$ be our homeomorphic map. By Lemma 1.16 , we obtain a sequence $n_{i}$ such that ${ }_{n_{i}} \mathcal{u}_{i}$, for all $i$ greater than some integer $N_{1}$, contains $[p]^{-}$. Let $\delta^{n}$ be a spherical neighborhood about $p^{\beta_{0}}$ of diameter $\pi_{0}=$ minimum of the distance of $p^{\beta_{0}}$ to the boundary of $\left(S \times I^{n-2}\right)^{\prime}$ 
and $\pi_{0} / 4$. Let $T$ be any arc contained in $\delta^{n} \cap\left(P \times I^{n-2}\right)^{\prime}$ having exactly the point $p^{\beta_{0}}$ in common with $[p]^{-}$. Take $q$, any point on $T$ distinct from $p^{\beta_{0}}$. Let $\pi_{2}$ be the distance from $p^{\beta_{0}}$ to $q$. By uniform continuity, there exists an
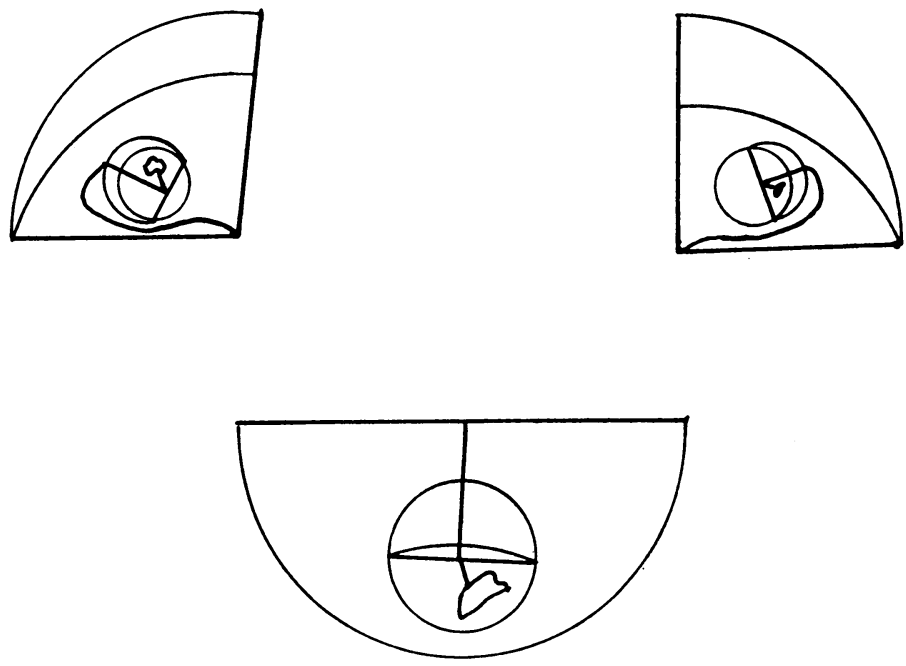

Figure 16. Cases 2, 3 and 4 of Lemma 1.15

$N_{2}$, such that $\pi\left([p]^{-},\left[J_{i, n_{i}}\right]^{-}\right)<\pi_{2}$ for all $i \geqq N_{2}$. Here, we take $N=\max \left(N_{1}, N_{2}\right)$. Then $J=\left[J_{N, n_{N}}\right]^{-}$separates $V$ and $T$. Hence, the arc $T$ has a point in common with $J$. This gives rise to a contradiction.

Theorem 1.3. Let $S$ be one of the two Claytor curves. Then, $S \times I^{n-2}, n \geqq 2$, cannot be imbedded in an n-manifold.

Proof. The only two cases are accounted for in Theorem 1.6 and Theorem 1.12 when the same arguments are applied to an Euclidean neighborhood of the homeomorph of the point $p^{\beta_{0}}$ in the $n$-manifold.

\section{Part II}

\section{Applications}

The Claytor Curves. Claytor [5] has proved the following theorem:

THEOREM 2.1. A necessary and sufficient condition that a Peano continuum be homeomorphic to a subset of the sphere, $S^{2}$, is that it contains neither a primitive skew curve nor a topological image of one of the Claytor curves.

Theorem 2.2. A necessary and sufficient condition that a Peano continuum $P$ containing no local cut points be imbeddable in a 2-manifold is that $P$ does not contain one of the Claytor curves. 
The sufficiency was proved by Moise and Young [10]. The necessity follows from the Main Theorems.

We shall observe (see Lemma 2.7) that a sequence of primitive skew curves converging to a point cannot be imbedded in a 2-manifold. If a Peano continuum $P$ is taken to contain no Claytor curve but $P$ does contain this sequence, then $P$ necessarily contains local cut points by Theorem 2.2.

More recently, Jones and Young [7] have proved the following

Theorem 2.3. Let $A_{1}, A_{2}, \cdots, A_{n}$ be nondegenerate connected locally compact sets. Suppose $P=A_{1} \times A_{2} \times \cdots \times A_{n}$ can be imbedded in an n-manifold $M^{n}$. Then each $A_{i}$ is an arc, a simple closed curve, a ray, or an open curve.

We use the Main Theorem and the theorems above in the following applications:

Theorem 2.4. A necessary and sufficient condition that the Cartesian product of $I^{n-2}, n \geqq 2$, and a Peano continuum $S$ which is void of primitive skew curves be imbeddable in an n-manifold is that $S$ be the topological image of a subset of the sphere $S^{2}$.

Proof. If $S$ is the topological image of a subset of the sphere, then $S \times I^{n-2}, n \geqq 2$, can be imbedded in $E^{n}$ (and, hence, an $n$-manifold) since this could happen if $S$ were the sphere itself.

For the converse, we only remark that, by the Main Theorem, $S$ cannot contain a Claytor curve and since $S$ contains no primitive skew curve, then it follows from Theorem 2.1 that $S$ is the topological image of a subset of the sphere.

TheOREM 2.5. A necessary and sufficient condition that the Cartesian product of $I^{n-2}, n \geqq 2$, and a Peano continuum $S$ which contains no local cut points be imbeddable in an n-manifold is that $S$ can be imbedded in a 2-manifold.

Proof. By the Main Theorem, $S$ does not contain a Claytor curve and since $S$ contains no local cut points, then, by Theorem $2.2, S$ can be imbedded in a 2-manifold.

For the converse, since $S$ can be imbedded in a 2-manifold, $M^{2}$, and since $I^{n-2}$ is an $(n-2)$-manifold, then $M^{2} \times I^{n-2}$ is an $n$-manifold containing a homeomorph of $S \times I^{n-2}$.

TheOREM 2.6. Let $A_{1}, A_{2}, \cdots, A_{n-2}$ be nondegenerate locally compact connected sets. Suppose $T=A_{1} \times A_{2} \times \cdots \times A_{n-2}$ can be imbedded in an $(n-2)$ manifold. Then, a necessary and sufficient condition that the Cartesian product of $T$ and a Peano continuum $S$ which contains no local cut points be imbeddable in an $n$-manifold is that $S$ be imbeddable in a 2-manifold.

Proof. By Theorem 2.3, $T$ contains a homeomorph of $I^{n-2}$. By the Main Theorem, $S$ contains no Claytor curve. Since $S$ contains no local cut points, we apply Theorem 2.2 to get our desired conclusion. 
On the other hand, if we imbed $T$ in the $(n-2)$-manifold $M^{n-2}$ and $S$ in the 2-manifold $M^{2}$, then $M^{n-2} \times M^{2}$ is an $n$-manifold containing a homeomorph of $T \times S$.

Lemma 2.7. If a Peano continuum $P$ contains an infinite sequence $S_{i}$ of primitive skew curves with diameters converging is a point $p$, then $P \times I^{n-2}$, $n \geqq 2$, cannot be imbedded in $E^{n}$.

Proof. We need only prove the Lemma for $n \neq 2$ since the lemma for the case $n=2$ follows immediately from the case $n=3$.

Suppose the contrary and let $\theta$ be our homeomorphic map. By the uniform continuity of $\theta$, we can choose an $\epsilon$ as we did for the Claytor curves and define $[W]-$ as before.

Let $N$ be the smallest positive integer such that any Jordan curve in $S_{i}$ is of diameter less than $\epsilon$ for all $i \geqq N$. It follows that for each Jordan curve $J_{j}$ in $S_{N},\left[J_{j}\right]$ - separates the space $v$ between $\Pi_{1}$ and $\Pi_{3}$ into two separated sets, $\mathfrak{u}_{j}$, bounded, and $\mho_{j}$, unbounded, such that one contains no points of $\left[S_{N}\right]^{-}$and the other contains all points of $\left[S_{N}\right]^{-}-\left[J_{j}\right]^{-}$. We can assume that $S_{N}$ is a primitive skew curve of type 1 since the argument is similar for the primitive skew curve of type 2. We consider the subset $S$ of $S_{N}$ consisting of the arcs $a x, a y, a z, b x, b y, b z, c x$, and $c z$. The set $S$ can be imbedded in the plane by a homeomorphic map $\theta^{*}$. There exists a 1-to-1 correspondence between the separations of the plane by $\theta^{*}(S)$ and the separations of $v$ by $[S]^{-}$. Let

$$
\begin{aligned}
& J_{1}=a x b z, \\
& A_{1}=a x b, \\
& A_{2}=x c z,
\end{aligned}
$$

and

$$
A_{3}=c y
$$

We consider only two cases since other cases are similarly argued.

Case 1. $\mathcal{u}_{j}$ contains $\left[A_{1}\right]^{-}-\left[J_{1}\right]^{-}$. Then $v_{j}$ contains the set $\left[A_{2}\right]^{-}-\left[J_{1}\right]-$. Since $[c]-$ is in $v_{j}$ and $[y]^{-}$in $\mathcal{u}_{j}$, then $\left[A_{3}\right]^{-}$is separated by $\left[J_{1}\right]^{-}$. But this is impossible and a contradiction arises.

Case 2. $v_{j}$ contains $\left[A_{1}\right]^{-}-\left[J_{1}\right]^{-}$. Then $u_{j}$ contains the set $\left[A_{2}\right]^{-}-\left[J_{1}\right]^{-}$. Since $[c]^{-}$is in $\mathcal{u}_{j}$ and $[y]^{-}$in $\mathcal{v}_{j}$, then $\left[A_{3}\right]^{-}$is separated by $\left[J_{1}\right]^{-}$. This also is impossible and a contradiction arises.

Theorem 2.8. A sufficient condition that a Peano continuum $P$ be locally imbeddable in the plane is that $P \times I^{n-2}$ be imbeddable in $E^{n}$ for some $n \geqq 2$. (If $P$ contains no local cut points, then by Theorem 2.2, the condition is also necessary.) 


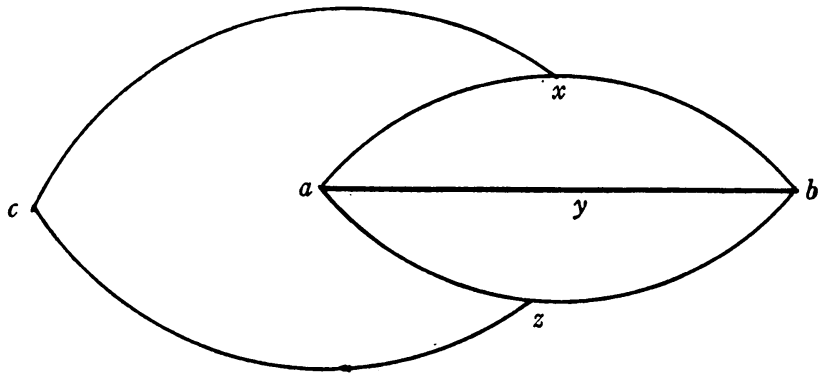

Figure 17. Set $\theta^{*}(S)$ of Lemma 2.7

Proof. Since $P \times I^{n-2}$ is imbeddable in $E^{n}$ for some $n \geqq 2$, then $P$ does not contain a Claytor curve by the Main Theorem. The set $P$ does not contain an infinite sequence of primitive skew curves converging to a point $p \in P$ by the preceding lemma. Hence, for any $p \in P$, there is a neighborhood of $p$ which contains neither a primitive skew curve nor one of the Claytor curves. By Theorem 2.1, this neighborhood is homeomorphic to a subset of the sphere $S^{2}$ and a neighborhood of $p$ is imbeddable in the plane.

Theorem 2.9. If a Peano continuum $P$ does not contain one of the Claytor curves and has no local cut points, then $P$ does not contain a sequence of primitive skew curves converging to a point $p \in P$.

Proof. We apply Theorem 2.2 and Lemma 2.7 .

Definition 2.10. A space $S$ is said to be of dimension $m$ (in the imbedding sense) if $m$ is the smallest non-negative integer such that, for some $n \geqq m$, $S \times I^{n-m}$ can be imbedded in $E^{n}$ (or an $n$-manifold).

Definition 2.11. A point $p$ of $S$ is said to be of dimension $m$ in $S$ if $m$ is the smallest non-negative integer such that there exists a neighborhood $V_{p}$ of $p$ such that $p \in V_{p}$ and $V_{p}$ is of dimension $m$.

Examples:

(1) Any point $p$ is of dimension 0.

(2) A Claytor curve is of dimension 3.

(3) A primitive skew curve is of dimension 2.

(4) A set $S$ consisting of sequence of primitive skew curves converging to a point $p$ in $S$ has dimension $\geqq 3$ at the point $p$.

(5) A Claytor curve $C$ has one point $p \in C$ of dimension 3 in $C$, a denumerable set of points of dimension 2 in $C$ and uncountably many points of dimension 1 in $C$.

(6) The base of a Claytor curve (See Definition 1.5) is of dimension 2.

Theorem 2.12. A necessary and sufficient condition that a Peano continuum be locally imbeddable in the plane is that $P$ contains no point of dimension $>2$. 
Proof. Sufficiency. Since $P$ has no point of dimension $>2$, then $P$ contains no Claytor curve by the Main Theorem. By Lemma $2.7, P$ contains no sequence of p.s.c. converging to a point $p \in P$. Hence, for every point $p \in P$, there is a neighborhood of $p$ which contains neither a p.s.c. nor one of the Claytor curves. By Theorem 2.1, this neighborhood is homeomorphic to a subset of the sphere $S^{2}$. Since, without loss of generality, we can assume this subset to be a proper subset of $S^{2}$, a neighborhood of $p$ is imbeddable in the plane.

Necessity. If $S$ is locally imbeddable in the plane, then every point $p \in S$ has a neighborhood $V_{p}$ of $p$ such that $p \in V_{p} \subset S$ and $V=V_{p} \times I^{3-2}$ can be imbedded in $E^{3}$ implies $p$ is of dimension no greater than 2 .

\section{REFERENCES}

1. R. H. Bing, $A$ decomposition of $E^{3}$ into points and tame arcs such that the decomposition space is topologically different from $E^{3}$, Ann. of Math. 65 (1957), 484-500.

2. - The Cartesian product of a certain nonmanifold and a line is $E^{4}$, Bull. Amer. Math. Soc. 64 (1958), 82-84.

3. K. Borsuk, On the decomposition of a locally connected compactum into Cartesian product of a curve and a manifold, Fund. Math. 40 (1953), 140-159.

4. W. W. S. Claytor, Topological immersion of Peanian continua in a spherical surface, Ann. of Math. 35 (1934), 809-835.

5. - Peano continua not imbeddable in a spherical surface, Ann. of Math. 38 (1937), 631-646.

6. R. H. Fox, On a problem of S. Ulam concerning Cartesian products, Fund. Math. 34 (1947), 278-287.

7. F. B. Jones and G. S. Young, Product spaces in n-manifolds, Proc. Amer. Math. Soc. 10 (1959), 307-308.

8. E. E. Moise, An indecomposable plane continuum which is homeomorphic to each of its nondegenerate subcontinua, Trans. Amer. Math. Soc. 63 (1948), 581-594.

9. - Remarks on the Claytor imbedding theorem, Duke Math. J. 19 (1952), 199-202.

10. E. E. Moise and G. S. Young, On imbedding continuous curves in 2-manifolds, Bull. Amer. Math. Soc. 54 (1948), 77.

11. R. L. Moore, Concerning triods in the plane and the junction points of plane continua Proc. Nat. Acad. Sci. U.S.A. 14 (1928), 85-88.

12. J. H. C. Whitehead, On the homotopy type of manifolds, Ann. of Math. 41 (1940), 825.

13. G. S. Young, Jr., A generalization of Moore's theorem on simple triods, Bull. Amer. Math. Soc. 50 (1944), 714.

Michigan State University, OAKLand, ROCHESTER, Michigan 